\title{
Intestinal immunopathology is associated with decreased CD73-generated adenosine during lethal infection
}

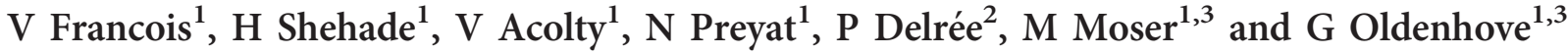

The ectonucleotidases CD39 and CD73 sequentially degrade the extracellular ATP pool and release immunosuppressive adenosine, thereby regulating inflammatory responses. This control is likely to be critical in the gastrointestinal tract where high levels of ATP are released in particular by commensal bacteria. The aim of this study was therefore to evaluate the involvement of the adenosinergic regulation in the intestine of mice in steady-state conditions and on acute infection with Toxoplasma gondii. We show that both conventional (Tconv) and regulatory (Treg) $\mathrm{CD}^{+}{ }^{+} \mathrm{T}$ lymphocytes express CD39 and CD73 in the intestine of naive mice. CD73 expression was downregulated during acute infection with $T$. gondii, leading to impaired capacity to produce adenosine. Interestingly, the expression of adenosine receptors was maintained and treatment with receptor agonists limited immunopathology and dysbiosis, suggesting that the activation of adenosine receptors may constitute an efficient approach to control intestinal inflammation associated with decreased ectonucleotidase expression.

\section{INTRODUCTION}

The CD39/CD73 pathway has gained increasing interest due to its dual role in the control of inflammation in vivo. ${ }^{1-4}$ Indeed, the ectoenzymes hydrolyze ATP and generate adenosine, thereby converting a pro-inflammatory stimulus into an antiinflammatory mediator (for review, see ref. 5).

ATP can be released from injured, ${ }^{6}$ dying or activated mammalian cells, ${ }^{7,8}$ degranulated platelets, ${ }^{9}$ or secreted by bacteria. ${ }^{10,11}$ More recently, an active mechanism of ATP secretion has been described that involves caspaseand pannexin 1 channels-dependent lysosomal exocytosis. ${ }^{8}$ Extracellular ATP binds to G-protein-coupled P2Y receptors (thereby activating the phopholipase $\mathrm{C} /$ inositol phosphate pathways or the protein kinase A pathway), and to trimeric ion channel P2X receptors which include P2X7, an activator of NLRP3 inflammasomes (for review, see ref. 12). ATP is an extracellular inflammatory mediator, which acts as a chemotactic signal for neutrophils, ${ }^{13}$ macrophages, and immature dendritic cells. ${ }^{14,15}$ A positive feedback loop has been described in the gastrointestinal tract whereby the activation of mast cells by ATP through P2X7 induces the production of inflammatory cytokines, chemokines, and leukotrienes, which recruit neutrophils and reinforce the inflammatory reaction. ${ }^{16}$ Importantly, ATP also acts as a costimulatory signal for $\mathrm{T}$ cells, ${ }^{17,18}$ thereby regulating the development of helper and regulatory T lymphocytes. ${ }^{19}$

The conversion of extracellular ATP to adenosine requires the membrane-bound ectonucleotidases CD39 and CD73 and counteracts directly (by triggering specific receptors) and indirectly (by decreasing the amount of extracellular ATP) the inflammatory action of ATP. Adenosine activates four $\mathrm{G}$-coupled receptors that decrease (A1 and $\mathrm{A} 3$ ) or increase (A2A and A2B) the levels of intracellular cyclic AMP (for review, see ref. 20). Adenosine has multiple actions aimed at maintaining tissue homeostasis and mediates its immunosuppressive effects mainly through the $\mathrm{A} 2 \mathrm{~A}$ and $\mathrm{A} 2 \mathrm{~B}$ receptors. ${ }^{20}$ The high-affinity receptor $\mathrm{A} 2 \mathrm{~A}$ and the low-affinity $\mathrm{A} 2 \mathrm{~B}$ receptor are expressed on most immune cells as well as endothelial and epithelial cells. A few reports indicate that adenosine suppresses both innate and adaptive responses.

\footnotetext{
'Department of Molecular Biology, Laboratory of Immunobiology, Université Libre de Bruxelles, Gosselies, Belgium and ${ }^{2}$ Institut de Pathologie et Génétique, Gosselies, Belgium. Correspondence: G Oldenhove (guillaume.oldenhove@ulb.ac.be)

${ }^{3}$ Authors share co-senior authorship.
} 
Adenosine has been shown to downmodulate the function of antigen-presenting-cells, ${ }^{21-23} \mathrm{~T}$ helper cells, and cytotoxic $\mathrm{T}$ lymphocyte ${ }^{24-26}$ and to potentiate the immunosuppressive role of regulatory $\mathrm{T}$ cells. ${ }^{27}$

In the gastrointestinal tract, commensal bacteria appear as a major source of intestinal luminal ATP ${ }^{11}$ suggesting that the CD39/CD73 pathway may have a prominent regulatory role aimed at maintaining homeostasis while controlling infections. Although the expression of CD39/CD73 has been first reported on regulatory $\mathrm{T}$ cells, ${ }^{28-30}$ recent reports suggest that primed uncommitted Th17 and T follicular helper cells also express $\mathrm{CD} 73,{ }^{31,32}$ raising the question of the role of this pathway in the control of immunity vs. tolerance in vivo.

In this study, we sought to determine the role of the CD39/CD73 pathway in a highly Th1 cell polarized microenvironment triggered by $T$. gondii infection and regulatory T-cell conversion. We characterized the immune status in the lamina propria (LP), intestinal epithelium, mesenteric lymph nodes (MLN), and in the spleen of infected mice and evaluated the outcome of adenosine receptor activation on infection, immunopathology, and intestinal homeostasis.

\section{RESULTS}

\section{Increased expression of CD39/CD73 on intestinal tconvs} and tregs correlates with a higher capacity to generate adenosine

We first examined the expression of the ectonucleotidases CD39 and CD73 by conventional $\mathrm{T}$ cells (Tconvs, CD4 ${ }^{+}$ Foxp $3^{-}$) and regulatory $\mathrm{T}$ cells (Tregs, $\mathrm{CD} 4{ }^{+} \mathrm{Foxp}^{+}$) in the spleen, the MLN, and the intestine of C57BL/6. Our data indicate that the expressions of CD39 and CD73 were higher in the small intestine, as compared with the spleen and MLN (Figure 1a). Although virtually all Tregs expressed CD73 and most Tregs expressed CD39, as expected, the proportion of $\mathrm{CD} 9^{+}$and $\mathrm{CD} 73^{+}$Tconvs was twofold higher in the LP and the intestinal epithelium as compared with the lymphoid organs. In addition, the level of expression of both molecules was much higher on $\mathrm{CD} 4^{+} \mathrm{T}$ cells purified from the $\mathrm{LP}$ and the epithelium of the small intestine (intraepithelial lymphocytes, IEL; Figure 1b).

A quantitative high-performance liquid chromatography (HPLC) assay was used to measure the production of adenosine by $\mathrm{CD} 4^{+}$T lymphocytes cultured in vitro in the presence of AMP. Tconvs from the LP generated more adenosine than
Tconvs from the spleen and MLN and this production was strictly dependent on CD73 (Figure 1c). These observations suggest that most $\mathrm{CD} 4^{+} \mathrm{T}$ lymphocytes (expressing or not Foxp3) in the small intestine have the capacity to produce adenosine. The role of CD73 in adenosine production was further highlighted by the twofold decrease in adenosine concentration in the sera of CD73 knockout (KO) mice as compared with wild-type (WT) mice in steady-state conditions (Figure 1d).

Interestingly, the analysis of CD39 and CD73 expression on $\mathrm{CD}^{+}{ }^{+} \mathrm{T}$ lymphocytes from spleen, $\mathrm{LP}$, and lung indicated that their enhanced expression was associated specifically with the gastrointestinal environment and not with T-cell activation in tissue sites in vivo, as the expressions of both ectonucleotidases were much higher in the LP than in the lung (Supplementary Figure 1a,b online).

\section{TGF- $\beta$ and retinoic acid promote CD73 expression}

Among the factors that may induce CD73 expression in the small intestine, transforming growth factor- $\beta$ (TGF- $\beta$ ) and retinoic acid (RA) were good candidates as they are constitutively present at high levels. In addition, TGF- $\beta$ has been shown to upregulate CD73 in vitro. ${ }^{33}$ Indeed, the expression of CD73 was strongly decreased on Tconvs and Tregs from CD4cre TGF- $\beta$ RII ${ }^{\text {lox/lox }}$ mice (Figure 2a). To investigate the role of RA expression, we used the well-known model of vitamin A-deficient mice. ${ }^{34}$ CD73 expression by Tconvs was significantly decreased in the spleen, MLN, and IEL but not in LP (Figure 2b). In contrast, virtually all Tregs expressed CD73 in vitamin A-sufficient or -deficient mice (Figure $2 \mathbf{b}$ ) and the intensity of expression remained unchanged (not depicted). Thus, RA synergized with TGF- $\beta$ only in Tconvs, a conclusion further supported by the increased expression of CD73 (Figure 2c) and the enhanced levels of phospho-Smad3 (Figure 2d) in Tconvs stimulated in vitro in the presence of TGF- $\beta$ and RA. We next tested various inhibitors of Smaddependent and Smad-independent pathways of TGF- $\beta$ signaling, and found that only SB431542, an inhibitor of Smad-dependent activation, prevented the increase in CD73 expression induced by the combination of TGF- $\beta$ and RA (Figure 2e). In contrast, CD39 expression was increased in CD4-cre TGF- $\beta$ RII ${ }^{\text {lox/lox }}$ mice, presumably due to spontaneous autoimmune disorder (Supplementary Figure 2a), and remained unchanged or slightly increased in vitamin A-deficient mice (Supplementary Figure 2b), indicating

\footnotetext{
Figure 1 Intestinal CD4 ${ }^{+}$Foxp3 $^{-}$Tlymphocytes have a high capacity to generate adenosine, in a CD73-dependent manner. (a, b) Cells were isolated from spleen, MLNs, LP, and epithelium of the small intestine (IEL) of C57BL/6, stained for TCR $\beta$, CD4, CD39, CD73, Foxp3 expression and analyzed by flow cytometry. (a) Data are expressed as dot plots of CD39 or CD73 and Foxp3 expression on TCR $\beta^{+} \mathrm{CD}^{+}{ }^{+}$-gated cells or (b) as the level of expression (mean fluorescence intensity, MFI) of CD39 or CD73 by CD73 ${ }^{+}$cells, among CD4 ${ }^{+}$Foxp3 ${ }^{-}$or CD4 ${ }^{+} \mathrm{Foxp}^{+}{ }^{+}$T lymphocytes. Data are representative of four independent experiments $(n=10)$. The non-parametric Kruskal-Wallis test followed by selected comparison by Dunn's multiple comparison tests was used. (c) $\mathrm{CD}^{+}{ }^{+} \mathrm{GFP}^{-}$and $\mathrm{CD} 4{ }^{+} \mathrm{GFP}^{+} \mathrm{T}$ lymphocytes were isolated from the spleen, MLN, and LP of WT or CD73KO-Foxp3 ${ }^{\mathrm{eGFP}}$ mice. Lymphocytes were incubated with AMP and the supernatants were tested for adenosine content by HPLC. Data are pooled from four independent experiments. Graph bars represent the median with interquartile range. (d) Amounts of adenosine were assessed by HPLC in sera from WT or CD73 mice. Data are pooled from three independent experiments $(n=13)$. The non-parametric Mann-Whitney $U$-test was used. $\left({ }^{\star} P<0.05\right.$, ${ }^{\star \star} P<0.01$, $\left.{ }^{\star \star \star} P<0.001\right)$. GFP, green fluorescent protein; HPLC, high-performance liquid chromatography; LP, lamina propria; MLN, mesenteric lymph node; WT, wild type.
} 

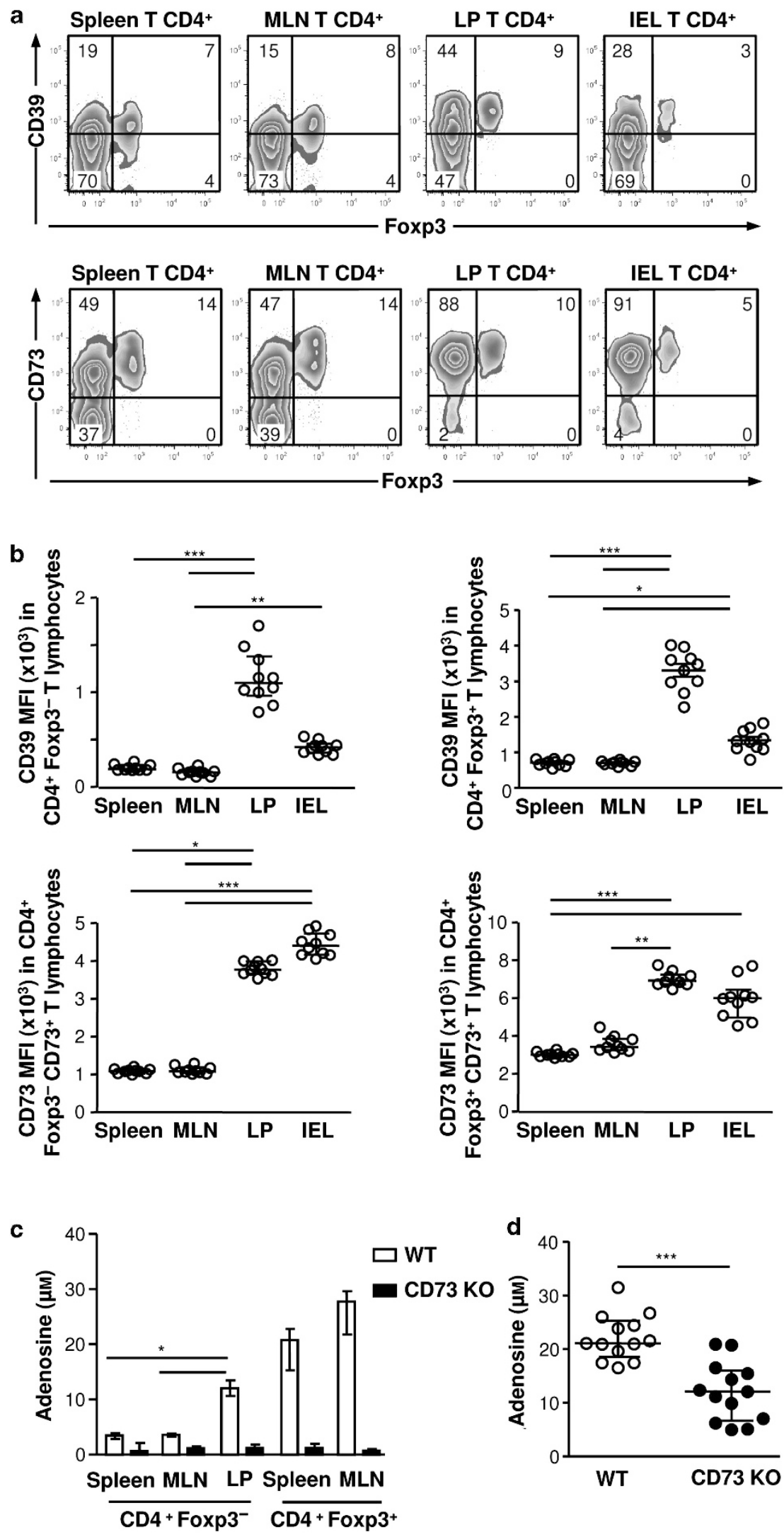


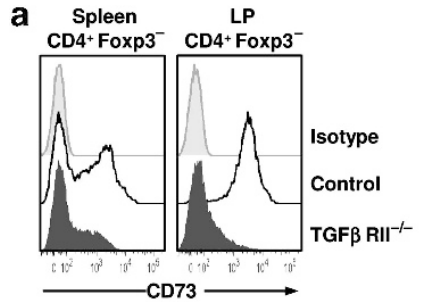

b

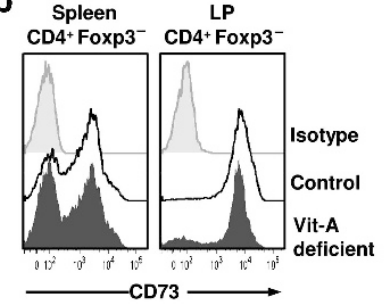

C

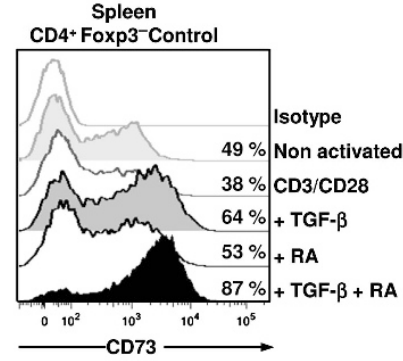

d

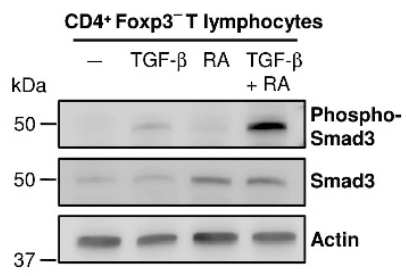

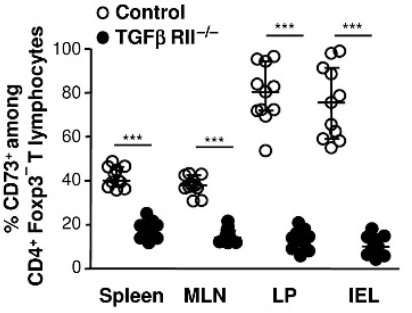
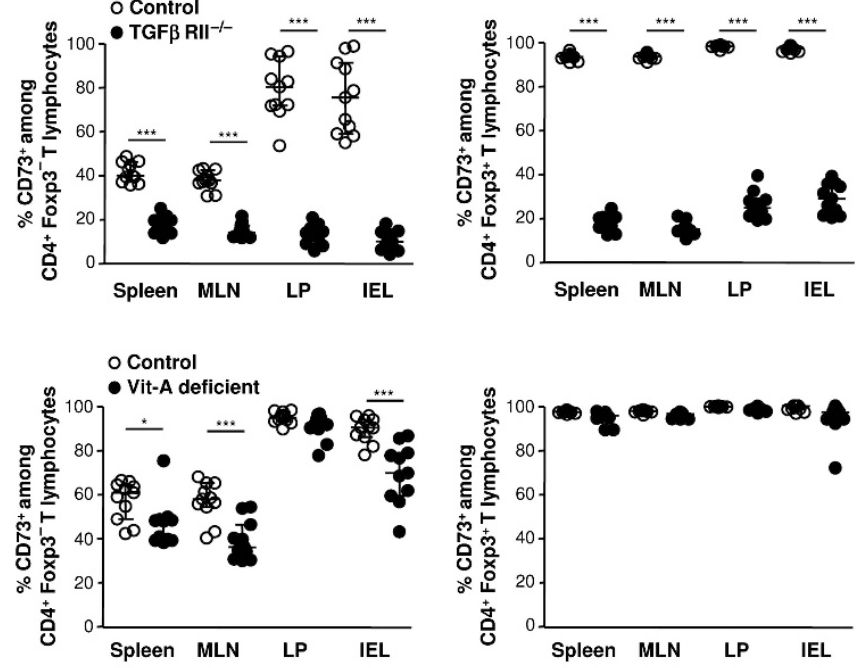

Spleen
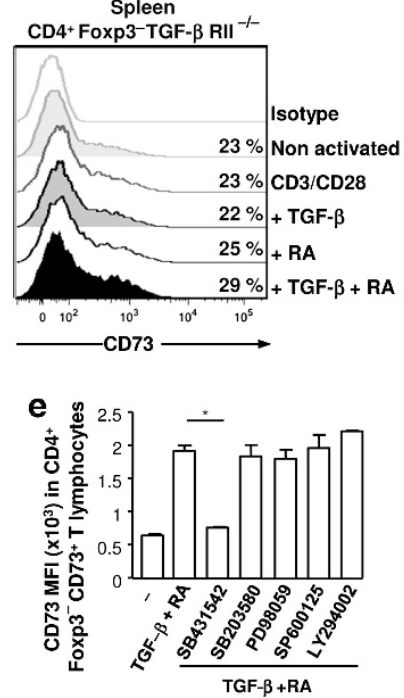

Figure 2 TGF- $\beta$ and retinoic acid promote CD73 expression. Cells were isolated from the spleen, MLN, LP, and IEL of (a) littermate control and CD4-cre TGF- $\beta$ RIIIox/lox mice or (b) control and vitamin A-deficient mice. Cells were stained for TCR $\beta$, CD4, CD73, and Foxp3 and analyzed by flow cytometry. Histograms show the expression of CD73 on CD4 ${ }^{+}$Foxp3 $^{-}$-gated cells. Graphs represent the percentage of $\mathrm{CD} 73^{+}$cells among $\mathrm{CD} 4{ }^{+}$ Foxp3 ${ }^{-}$T lymphocytes (left panels) or CD4 ${ }^{+}$Foxp3 $^{+}$T lymphocytes (right panels). Data are pooled from three independent experiments $(n=11)$. (c) $\mathrm{CD}^{+}{ }^{+} \mathrm{CD} 25^{-}$T lymphocytes were isolated from the spleen of littermate control and CD4-cre TGF- $\beta$ RII ${ }^{\text {lox/lox }}$ mice and stimulated with anti-CD3/CD28 in the absence or presence of TGF- $\beta$ and/or RA. $24 \mathrm{~h}$ later, TCR $\beta^{+} \mathrm{CD} 4^{+}$Foxp3 ${ }^{-}$-gated cells were analyzed for CD73 by flow cytometry. Histograms show the expression of CD73. The results are representative of three independent experiments. (d, e) $\mathrm{CD}^{+}{ }^{+} \mathrm{GFP}^{-} \mathrm{T}_{\text {lymphocytes were isolated from the }}$ spleen of Foxp3 ${ }^{\text {eGFP }}$ mice and were activated as in c. (d) After $24 \mathrm{~h}, \mathrm{CD}^{+} \mathrm{GFP}^{-}$T lymphocytes were sorted by flow cytometry. Cell lysates were analyzed with western blotting for phosphorylated Smad3 (p-Smad3) and Smad3. Actin served as a loading control. The results are representative of three independent experiments. (e) Cultures were supplemented with TGF- $\beta$ receptor kinase inhibitor (SB431542), p38 inhibitor (SB203580), MAPK/ ERK kinase inhibitor (PD98059), JNK inhibitor (SP600125) and PI3 kinase inhibitor (LY294002). Data are expressed as MFI of CD73 expression by $\mathrm{CD}^{+}{ }^{-} \mathrm{Foxp}^{-} \mathrm{CD}^{-} 3^{+} \mathrm{T}$ lymphocytes and are representative of three independent experiments. Graph bars represent the median with interquartile range. The non-parametric Mann-Whitney $U$-test was used $\left({ }^{\star} P<0.05,{ }^{* \star \star} P<0.001\right)$. GFP, green fluorescent protein; IEL, intraepithelial lymphocyte; LP, lamina propria; MFI, mean fluorescence intensity; MLN, mesenteric lymph node; RA, retinoic acid; TGF- $\beta$, transforming growth factor- $\beta$.

that neither TGF- $\beta$ nor RA was required for CD39 expression. This conclusion was supported by in vitro data showing that CD39 was not increased on addition of TGF- $\beta$ and/or RA (Supplementary Figure 3). Collectively, these data highlight the major role of TGF- $\beta$ as inducer of CD73 (but not CD39) expression by Tconvs and Tregs in the intestine. RA enhances the TGF- $\beta$-induced CD73 expression on Tconvs but appears dispensable for expression on Tregs.

\section{Reduced adenosine production parallels reduced CD73 expression during acute infection by $T$. gondii}

We next examined the role of adenosine during a highly Th1 cell type-polarized mucosal immune response. We used C57BL/6 mice infected orally with 40 cysts of the type II T. gondii strain, ${ }^{35,36}$ in which the acute infection was associated with breakdown of the Treg cell population, resulting from blockade of Treg induction and collapse of the endogenous 

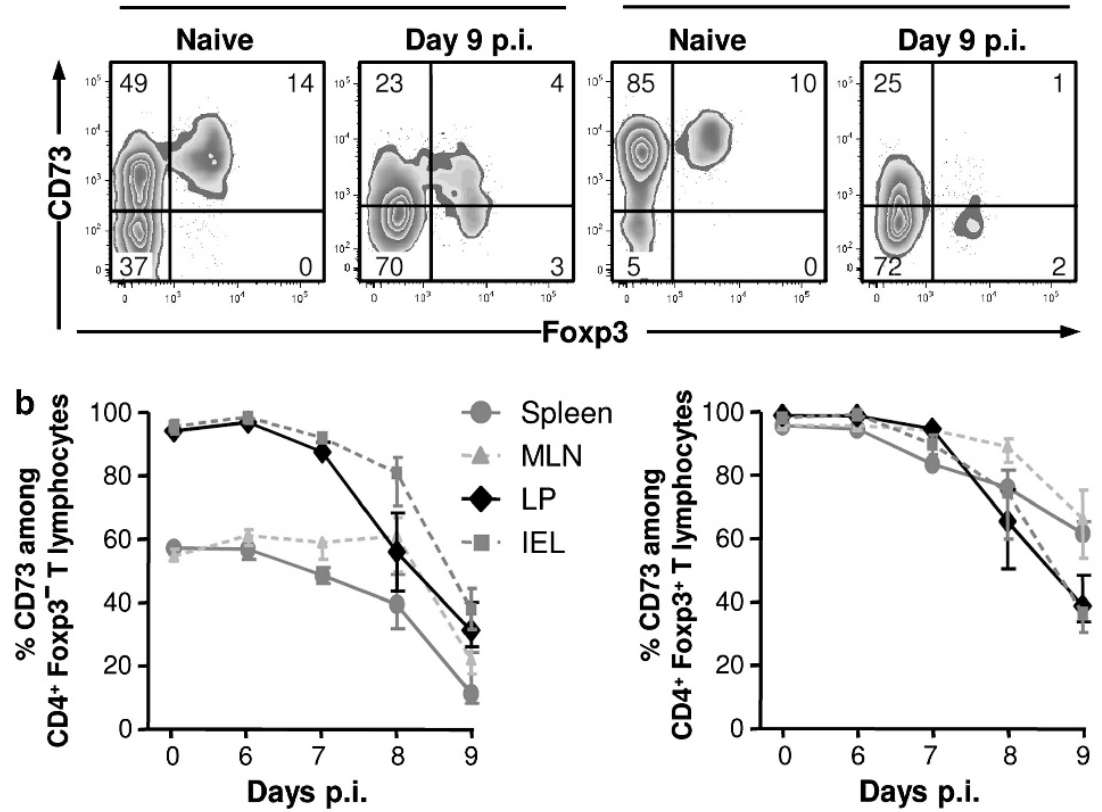

C

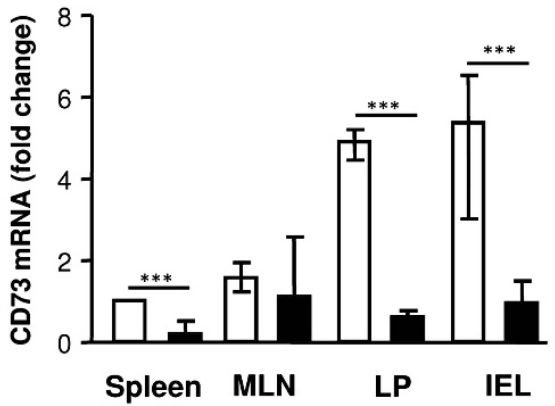

e

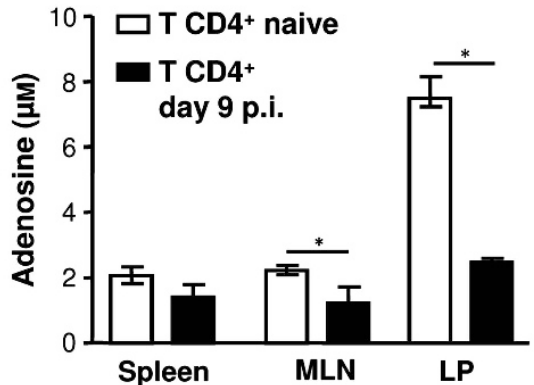

d
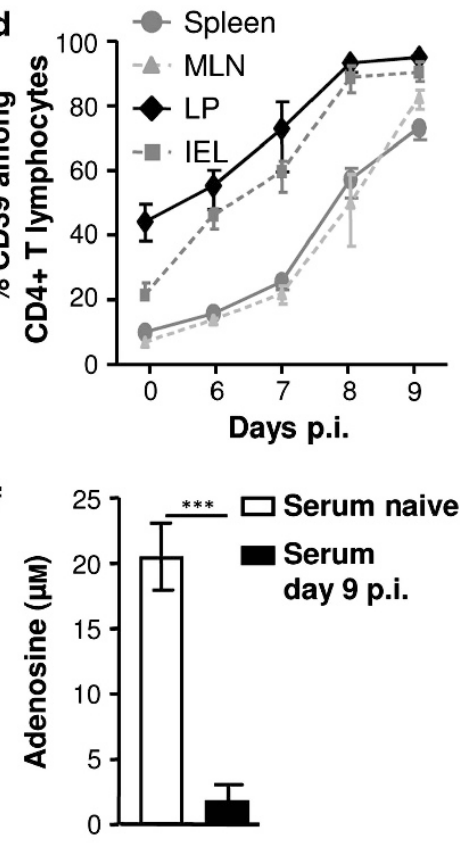

Figure 3 The generation of adenosine is compromised during the acute phase of toxoplasmosis. C57BL/6 mice were infected orally with 40 cysts of ME-49. Cells were isolated from the spleen, MLN, LP, and IEL, stained for TCR $\beta$, CD4, CD39, CD73, and Foxp3 and analyzed by flow cytometry. (a) Dot blots represent $\mathrm{CD} 73$ and Foxp3 expression by CD4 ${ }^{+} \mathrm{T}$ lymphocytes at day 0 or at day 9 post infection (p.i.) from spleen and LP compartments. (b) Percentages of TCR $\beta^{+} \mathrm{CD} 4^{+}$Foxp3 negative or positive cells expressing CD73 between days 0 and 9 after infection in spleen, MLN, LP, and IEL compartments. The results are representative of five independent experiments $(n=5)$. (c) At day 0 or at day 9 p.i., TCR $\beta^{+}$CD4 ${ }^{+}$lymphocytes from spleen, MLN, LP, or IEL were purified and mRNA extracted. Quantitative RT-PCR of the expression of CD73 mRNA was performed. Data are pooled from four independent experiments. (d) Percentages of TCR $\beta^{+} \mathrm{CD} 4^{+}$cells expressing CD39 between days 0 and 9 after infection in spleen, MLN, LP, and IEL compartments $(n=5)$. (e) TCR $\beta^{+}$CD4 ${ }^{+}$lymphocytes from spleen, MLN, LP, or IEL were purified at day 0 or at day 9 p.i. and incubated with AMP. The supernatants were collected and adenosine concentration was analyzed by HPLC. Data are pooled from four independent experiments. (f) Amounts of adenosine were assessed by HPLC in sera from naive or day 9 infected mice. Data are pooled from three independent experiments $(n=12)$. (c, e, $\mathbf{f})$. Graph bars represent the median with interquartile range. The non-parametric Mann-Whitney U-test was used to determine statistical differences $\left({ }^{\star} P<0.05,{ }^{* * *} P<0.001\right)$. HPLC, high-performance liquid chromatography; IEL, intraepithelial lymphocyte; LP, lamina propria; MLN, mesenteric lymph node; RT-PCR, reverse transcription-PCR. 
a
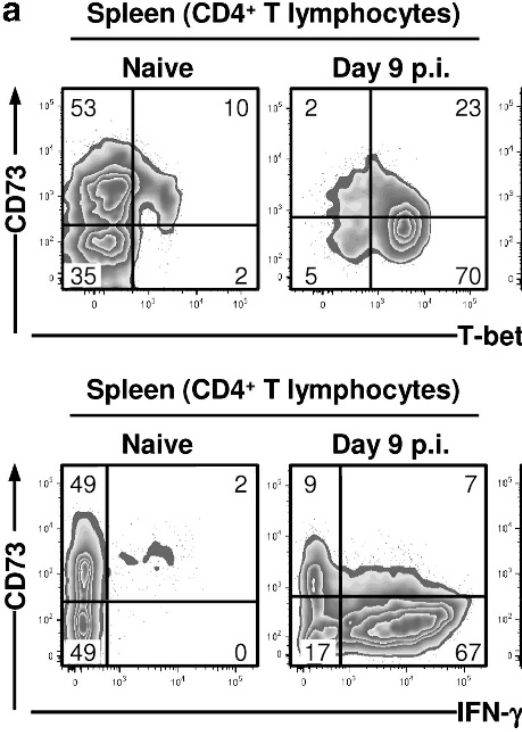

b

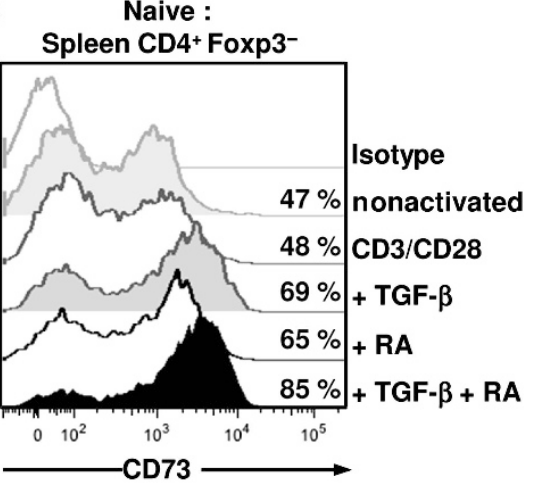

LP (CD4+ T lymphocytes)

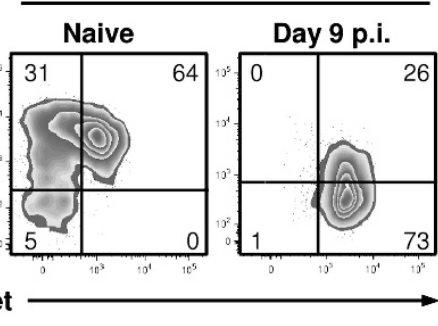

LP (CD4+ T Iymphocytes)

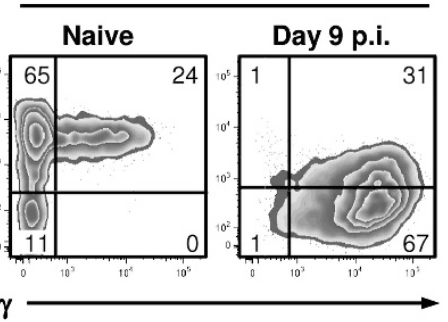

Day 9 p.i. : Spleen CD4+ Foxp3-

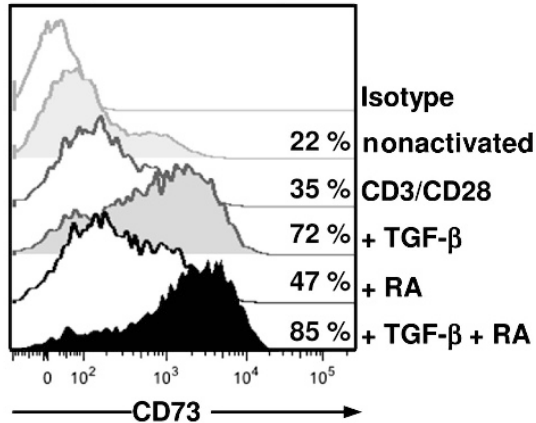

d

LP (CD4+ T lymphocytes)

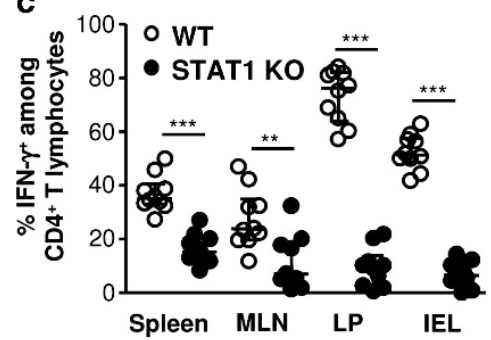

WT STAT1 KO

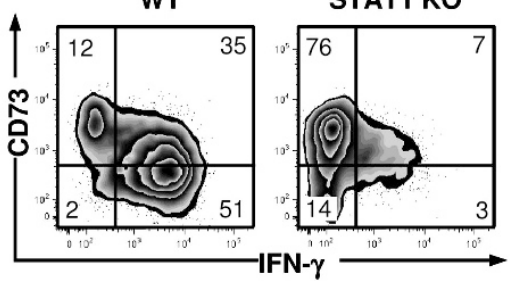

Figure 4 Role of TGF- $\beta$ and STAT1 in CD73 expression. Mice were inoculated orally with 40 cysts of ME-49. (a) Spleen and LP cells were isolated from C57BL/6 naive or day 9 infected mice. Cells were restimulated in vitro with PMA and ionomycin in the presence of Brefeldin A. Cells were stained for TCR $\beta$, CD4, T-bet, CD73, and IFN- $\gamma$ and analyzed by flow cytometry. Dot plots illustrate CD73, T-bet, and IFN- $\gamma$ staining profiles of TCR $\beta^{+}$CD4 ${ }^{+}$-cells. The results are representative of five independent experiments. (b) CD4 ${ }^{+} \mathrm{GFP}^{-} \mathrm{T}$ lymphocytes were isolated by cell sorting from naive (left panel) or infected (right panel) Foxp3 ${ }^{\text {eGFP }}$ mice and activated with anti-CD3/CD28 in the absence or presence of TGF- $\beta$ and/or RA. After 24 h, CD4 ${ }^{+}$GFP ${ }^{-}$T lymphocytes were analyzed for CD73 expression by flow cytometry. The results are representative of three independent experiments. (c) Graph summarizes the percentage of TCR $\beta^{+} \mathrm{CD} 4^{+}$cells producing IFN- $\gamma$ in the spleen, MLN, LP, and IEL compartments from WT and STAT1 KO infected mice. Data are pooled from three independent experiments $(n=10)$. (d) Dot plots illustrate CD73 and IFN- $\gamma$ staining profiles of CD4 ${ }^{+}$TCR $\beta^{+}$cells from WT or STAT1 KO mice. The non-parametric Mann-Whitney U-test was used to determine statistical differences $\left({ }^{\star \star} P<0.01\right.$, $\left.{ }^{\star * \star} P<0.001\right)$. GFP, green fluorescent protein; IEL, intraepithelial lymphocyte; IFN- $\gamma$, interferon- $\gamma$; KO, knockout; LP, lamina propria; MLN, mesenteric lymph node; RA, retinoic acid; TGF- $\beta$, transforming growth factor- $\beta$; WT, wild type.

Treg. We first monitored the expression of CD73 at various days after infection. The proportion of $\mathrm{CD}^{+} 3^{+}$cells strongly decreased from day 7 post infection (p.i.) among Tconvs and Tregs in the spleen, MLN, LP, and IEL (Figure 3a,b). The level of mRNA coding for CD73 was strongly reduced in the intestine (Figure 3c). In contrast, the proportion of $\mathrm{CD} 4^{+}$ $\mathrm{T}$ cells expressing the ectonucleotidase CD39 was increased in the same conditions (Figure 3d). The capacity to produce adenosine was lower in MLN and LP compartments 9 days p.i., as compared with untreated mice (Figure 3e), and the level of 

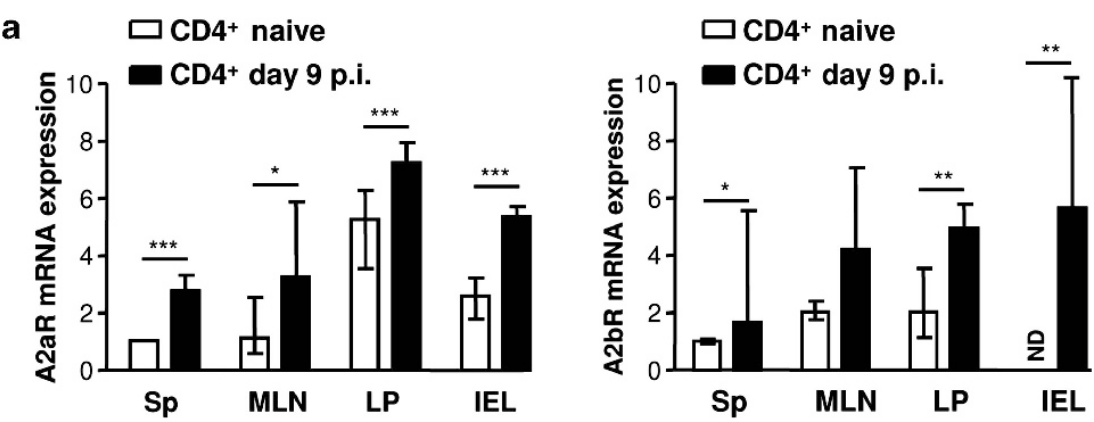

b

O Day 9 p.i.

- Day 9 p.i. : A2AR and A2BR agonists
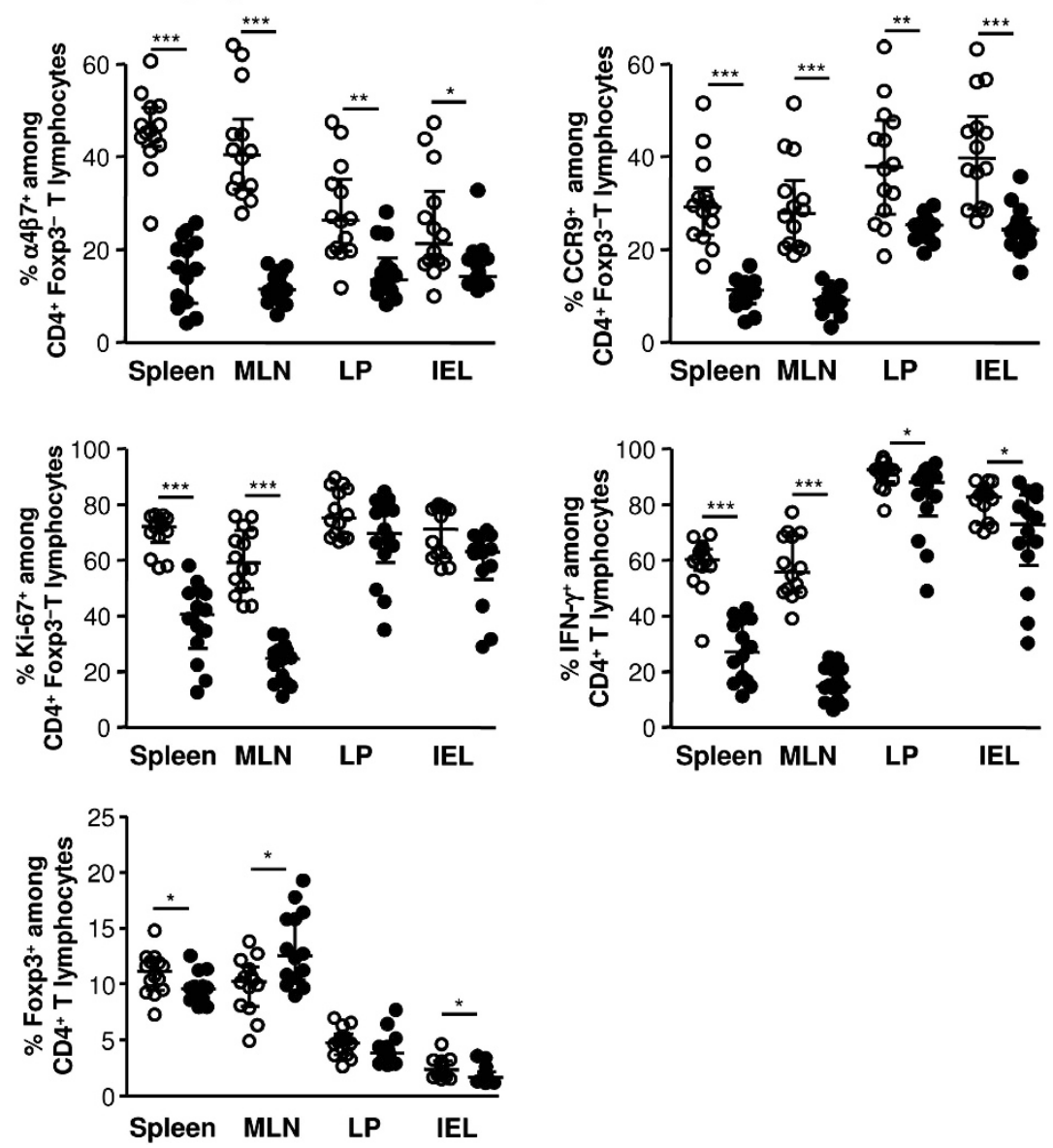

Figure 5 Activation of A2A and A2B receptors downregulates T-cell activation during acute toxoplasmosis. C57BL/6 mice were inoculated orally with 40 cysts of ME-49. (a) At day 9 after infection, TCR $\beta^{+}$CD4 ${ }^{+}$lymphocytes from spleen, MLN, LP, or IEL were cell-sorted. mRNA was extracted and quantitative RT-PCR of A2A and A2B receptors mRNA was performed. Data are pooled from four independent experiments. Graph bars represent the median with interquartile range. (b) At day 3 p.i., mice were treated daily with the combination of A2A and A2B receptor agonists or DMSO until day 9. Spleen, MLN, LP, and IEL cells were isolated and restimulated with PMA and ionomycin in the presence of Brefeldin A. Cells were stained for TCR $\beta$, CD4, $\alpha 4 \beta 7$, CCR9, Ki-67, IFN- $\gamma$, and Foxp3 and analyzed by flow cytometry. Data are pooled from three independent experiments $(n=14)$. The non-parametric Mann-Whitney $U$-test was used to determine statistical differences $\left({ }^{\star} P<0.05,{ }^{\star \star} P<0.01,{ }^{\star \star \star} P<0.001\right)$. DMSO, dimethyl sulfoxide; IEL, intraepithelial lymphocyte; LP, lamina propria; MLN, mesenteric lymph node; RT-PCR, reverse transcription-PCR.

adenosine measured in the serum of infected mice was reduced by about 13 -fold in infected mice (Figure $3 \mathbf{f}$ ).

The impaired capacity to generate adenosine, during the acute phase of toxoplasmosis, is likely to contribute to inflammation, as the Th1 phenotype, characterized by T-bet expression and interferon- $\gamma$ (IFN- $\gamma$ ) secretion, was correlated with decreased CD73 expression by $\mathrm{CD} 4^{+} \mathrm{T}$ lymphocytes in the spleen and LP (Figure 4a). Addition of TGF- $\beta$ (alone or in combination with RA) on Tconvs isolated from infected mice 9 days p.i. restored the expression of CD73 to control levels (Figure 4b), suggesting that decreased CD73 expression may result, at least in part, from a defect in TGF- $\beta$ production in 
a
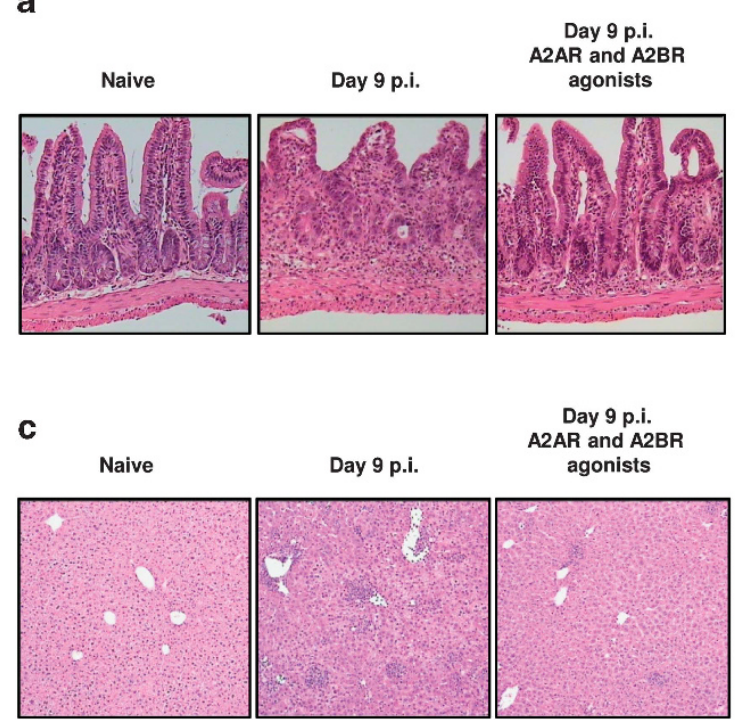

e

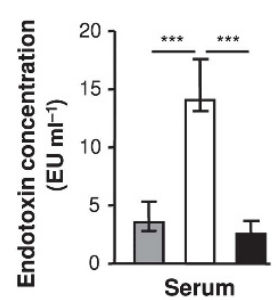

f

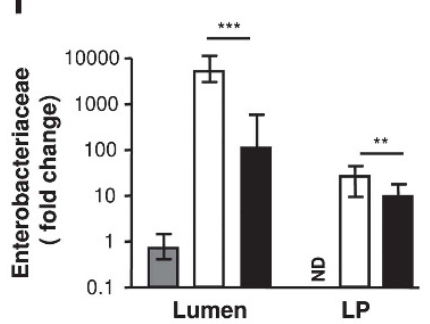

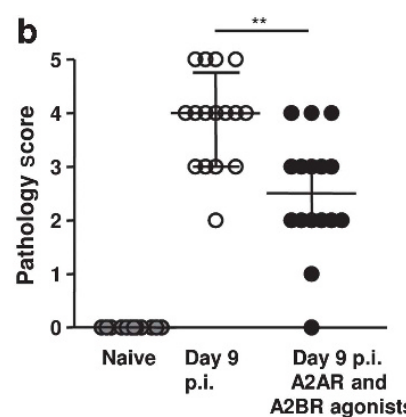

d
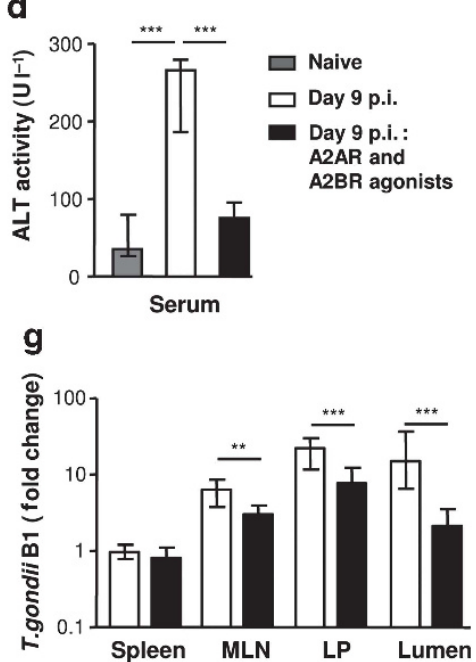

Figure 6 Activation of $\mathrm{A} 2 \mathrm{~A}$ and $\mathrm{A} 2 \mathrm{~B}$ receptors protected mice from pathology during acute toxoplasmosis. C57BL/6 mice were inoculated orally with 40 cysts of ME-49. A2AR and A2BR agonists were injected intraperitoneally from days 3 to 9 after infection. As a control, equivalent volume of DMSO was used. (a, c) Representative histological sections of the ileum and the liver from naive or infected mice treated or not with agonists (hematoxylin and eosin, magnification, $\times 100)$. (b) Pathology scores in the small intestine ileum. Data are pooled from four independent experiments ( $n=16)$. (d, e) Amounts of hepatic alanine aminotransferase and endotoxin were assessed in sera. (f) Quantitative PCR analysis in the lumen and lamina propria of small intestine of Enterobacteriaceae. (g) Quantification of parasite load by qPCR analysis in the spleen, MLN, LP, and lumen of B1 gene. (d-g). Data are pooled from three independent experiments $(n=12)$. Graph bars represent the median with interquartile range. The non-parametric Mann-Whitney $U$-test was used to determine statistical differences $\left({ }^{\star} P<0.05,{ }^{* \star} P<0.01,{ }^{* * *} P<0.001\right)$. DMSO, dimethyl sulfoxide; LP, lamina propria; MLN, mesenteric lymph node.

infected mice (see Discussion). Finally, as inflammatory cytokines have been shown to downregulate CD73 expression in vitro (see Discussion), we monitored CD73 expression in infected WT and STAT1 KO mice. As expected, CD4 ${ }^{+} \mathrm{T}$ lymphocytes displayed impaired IFN- $\gamma$ expression (Figure 4c), which resulted in uncontrolled tachyzoite expansion (Supplementary Figure 4). The proportion of $\mathrm{CD}^{+} \mathrm{T}$ lymphocytes expressing CD73 in the LP remained elevated 8 days p.i. (Figure $4 \mathbf{d}$ ). Collectively, these observations reveal a defect in adenosine production on T. gondii acute infection, which could result from altered TGF- $\beta$ and/or IFN- $\gamma$ production.

\section{Activation of A2A and A2B receptors protects mice from pathology}

To gain further insight into the role of adenosine as an anti-inflammatory agent in the intestinal tract, we treated mice with agonists of adenosine receptor(s). As a prerequisite, we monitored the expression of mRNA coding for A2A and $\mathrm{A} 2 \mathrm{~B}$ receptors during infection. The data in Figure 5a show that mRNAs coding for both receptors were expressed on $\mathrm{CD} 4{ }^{+} \mathrm{T}$ lymphocytes in the spleen and the intestine 9 days p.i. The mRNA expression remained unchanged or even increased. Administration of $\mathrm{A} 2 \mathrm{~A}$ and $\mathrm{A} 2 \mathrm{~B}$ receptor agonists resulted in the downregulation of gut-homing molecules, decreased proliferation (although not statistically significant in LP and IEL) and reduced IFN- $\gamma$ expression by $\mathrm{CD} 4{ }^{+}$ Tconvs in spleen and MLN, and to a lesser extent in LP and IEL (Figure 5b), on T. gondii infection. In contrast, the proportion of Tregs was increased in the MLN and slightly reduced or unchanged in spleen, LP, and IEL (Figure 5b, lower panel). Noteworthy, these anti-inflammatory effects translated into amelioration of disease, as assessed by decreased pathology in gut and liver (Figure 6a-d and Supplementary Figure 5), reduced dysbiosis (Figure 6e, f), and decreased parasite load (Figure $\mathbf{6 g}$ ). These observations indicate that treatment with the combination of agonists downmodulated inflammatory reactions, gut dysbiosis, and tissue injury during $T$. gondii acute infection while controlling parasite burden. 


\section{DISCUSSION}

There is an increasing evidence that the intestinal microenvironment is a preferential site for the generation of adenosine. In particular, we show here that most $\mathrm{CD} 4^{+}$ T lymphocytes in the LP and among IEL, including Foxp3 ${ }^{-}$ Tconvs, express CD39 and CD73, the enzymes which sequentially dephosphosphorylate ATP to produce adenosine. These observations complete previous studies showing that mice lacking CD73 displayed increased colonic inflammation. ${ }^{3,37}$ Our data using CD4-cre TGF- $\beta$ RII ${ }^{\text {lox/lox }}$ mice and vitamin $A$-deficient mice indicate that TGF- $\beta$ and RA synergize to induce CD73 expression in Tconvs, whereas RA is dispensable for CD73 expression by Tregs. The source of RA is probably related to the population of $\mathrm{CD}_{103}{ }^{+}$dendritic cells in the $\mathrm{LP}^{38,39}$ and to the MLN dendritic cells and stromal cell expressing aldh $1 \mathrm{a} 2,{ }^{40}$ a retinal dehydrogenase involved in the conversion of retinal into RA. Thus, TGF- $\beta$ and RA are not only cofactors of Treg generation, as shown previously, ${ }^{39,41}$ but also favor the production of adenosine by Tconvs, thereby amplifying the immunosuppressive effect. Our observations strongly suggest that Tconvs themselves (and not newly converted Tregs) express elevated levels of $\mathrm{CD} 73$, as in vitro (Supplementary Figure 6) or in vivo (Figure $\mathbf{4 b}$ ) activated Th1 cells upregulated CD73 in the presence of TGF- $\beta$ and RA. The capacity of most intestinal CD $4^{+}$T lymphocytes (and probably other cell populations) to metabolize extracellular ATP produced at high levels by gut flora ${ }^{10,11}$ suggests that adenosine must be constitutively produced in the gut microenvironment.

We also show that the expression of CD73, which contributes for $50 \%$ of the adenosine measured in serum, was strongly diminished during acute $T$. gondii and that the regulation occurs at the transcriptional level. Notably, administration of $\mathrm{A} 2 \mathrm{~A} / \mathrm{A} 2 \mathrm{~B}$ receptors agonists in infected mice limited the immune pathology and mucosal damage, thereby highlighting the role of adenosine as an anti-inflammatory factor in the gastrointestinal tract. These data are in accordance with a report showing that activation of $\mathrm{A} 2 \mathrm{~A}$ adenosine receptor attenuated inflammation of the intestinal mucosa in rabbit colitis models and in a spontaneous mouse ileitis. ${ }^{42}$ Similarly, combination therapy with an adenosine $\mathrm{A} 2 \mathrm{~A}$ receptor agonist and alanyl-glutamine (which enhances intestinal repair) efficiently reverted Clostridium difficile toxin-A induced enteritis. ${ }^{43}$ In a murine model of Lyme disease, Yegutkin et al. ${ }^{44}$ reported enhanced adenosine generating capacities in resistant C57BL/6 mice, which correlated with decreased joint swelling. The protective effect of adenosine (or adenosine receptor activation) may involve several mechanisms, including (i) direct inhibition of effector cells, through IFN- $\alpha$ A-dependent induction of IL- $10^{37}$ or accumulation of intracellular cAMP, ${ }^{45}$ (ii) indirect inhibition of inflammation by increasing the number of Tregs and enhancing their immunoregulatory activity, ${ }^{46}$ (iii) participation to tissue repair. In addition, adenosine has been shown to reinforce the intestinal barrier, ${ }^{47,48}$ an effect that may be critical to limit intestinal inflammation during $T$. gondii infection.
The role of TGF- $\beta$ in immunoregulation has been amply documented. TGF- $\beta$ has been shown to favor the development of peripheral Tregs and sustain the suppressive function of thymic Tregs. More recently, Regateiro et al. ${ }^{33}$ have reported that TGF- $\beta$ upregulated CD73 mRNA expression in $\mathrm{CD} 4^{+} \mathrm{T}$ cells, dendritic cells, and macrophages in vitro, unless antagonistic proinflammatory cytokines predominated. Therefore, the downregulation of CD73 expression in T. gondii-infected mice may result from several factors including: (i) the breakdown of the Treg cell population; ${ }^{36}$ (ii) the highly polarized Th1-type immune response characterized by high levels of IFN- $\gamma$; (iii) the reduced production of TGF- $\beta$. Among inflammatory cytokines, IFN- $\gamma$ was the most efficient in antagonizing the induction of $\mathrm{CD} 73$ by TGF- $\beta$ in vitro. ${ }^{33}$ Similarly, our observations clearly point to a role of IFN- $\gamma$, as suggested by the unchanged CD73 expression by $\mathrm{CD}^{+}{ }^{+} \mathrm{T}$ cells from the LP in STAT-1-deficient mice during acute infection. In addition, the role of TGF- $\beta$ in CD73 downregulation was suggested indirectly by our data showing that exogenous TGF- $\beta$ strongly increased the expression of CD73 on spleen $\mathrm{CD} 4{ }^{+} \mathrm{T}$ cells from infected mice. Whether TGF- $\beta$ production is reduced in the gut on $T$. gondii infection is presently unclear and deserves further attention.

In addition to its beneficial role as an anti-inflammatory mediator, adenosine may be deleterious to the host by facilitating T. gondii differentiation to long-lived tissue cysts in the central nervous system. Indeed, Mahamed et al. ${ }^{49}$ have reported that $\mathrm{CD} 73^{-} \mathrm{KO}$ mice were less susceptible to Toxoplasmosis despite similar levels of parasite dissemination in the brain during the acute stage of infection and similar degree of leukocyte infiltration during chronic infection. The authors further showed that CD73 expression was required for the formation of cysts and that exogenous adenosine was able to rescue $T$. gondii cysts formation in CD73-deficient host cells. ${ }^{49}$ It is interesting to note that the transport of adenosine occurred across cell membranes independently of its receptors, thereby validating the beneficial effect of adenosine receptor agonists. ${ }^{49}$

The immunopathology associated with T. gondii infection has been well documented. Bacteria have been shown to escape the gut and translocate to the mesenteric LN, liver, and spleen. Raetz et al. have identified the sequence of events leading to dysbiosis characterized by uncontrolled expansion of bacteria of the Enterobacteriacae family. ${ }^{50} \mathrm{~T}$ cell-intrinsic MyD88 signaling by pathogen induced mucosal-polarized Th1-type immunity, which led to the elimination of Paneth cells, loss of lysozyme, and defensin expression and subsequent expansion of intestinal bacteria, in particular of the Enterobacteriacae family. ${ }^{50}$ Of note, T. gondii infection in germ-free mice did not lead to intestinal inflammation and loss of Paneth cells, underlying the role of the dysbiosis in the pathology. Another report associated the disruption of the intestinal barrier with T-cell immunity specific to commensal bacteria. ${ }^{51}$ Our own observations reveal a more than 5,000-fold expansion of Enterobacteriaceae in the lumen 9 days p.i., which was reduced to 300 -fold expansion on agonist treatment.

In conclusion, we show herein that adenosine production is impaired during T. gondii acute infection, which paralleled intestinal immunopathology associated with dysbiosis and 
disruption of the intestinal barrier. Activation of the adenosine $\mathrm{A} 2 \mathrm{~A}$ and $\mathrm{A} 2 \mathrm{~B}$ receptors not only downregulated inflammatory responses and attenuated tissue damage, but also reduced parasitic load, suggesting that adenosine may constitute an interesting target for immune intervention in the case of inflammatory bowel diseases.

\section{METHODS}

Mice. C57BL/6 (WT) mice were purchased from Harlan Nederland. CD73 KO were kindly provided by Dr Thompson (University of Oklahoma); Foxp $3^{\text {eGFP }}$ reporter mice and TGF- $\beta$ RII ${ }^{\text {lox/lox }}$ mice by Dr Liston (KUL, Leuven); STAT1 KO mice by Dr Goriely (ULB, Gosselies); CD4-Cre mice by Dr Van Loo (University of Gent). All mice were bred and maintained under pathogen-free conditions. Mice between 8 and 12 weeks of age were used.

Diet studies. For vitamin A deficiency experiments, vitamin A-deficient (TD.86143) and -sufficient (TD.93160) diets were purchased from Harlan Teklad Diets. At day 14 of gestation, females were administered either vitamin A-deficient or -sufficient diet and maintained on diet until weaning of litter. On weaning, females were returned to standard Harlan chow, whereas weanlings were maintained on special diet.

Parasite and infection protocol. ME-49 type II of T. gondii was kindly provided by Dr De Craeye (ISP, Belgium) and was used for the production of tissue cysts in C57BL/6 mice, which were inoculated 1-3 months previously with three cysts by gavage. Animals were killed, and the brains were removed. Tissue cysts were counted and mice were infected by intragastric gavage with 40 cysts.

Cell isolation/tissue preparation. Cells from spleen, MLN, LP, and IEL were prepared as described previously, ${ }^{38}$ with the following modifications for LP digestion: minced tissue was further digested with serum-free RPMI-1640 containing: $25 \mathrm{~mm}$ HEPES, $50 \mu \mathrm{m}$ $\beta$-mercaptoethanol, $100 \mu \mathrm{g} \mathrm{ml}^{-1}$ liberase TI (Roche, Vilvoorde, Belgium) and $500 \mu \mathrm{g} \mathrm{ml}^{-1}$ DNase I (Sigma, Diegem, Belgium). Cells were resuspended in complete RPMI-1640 medium containing 10\% fetal calf serum.

T-cell purification. Single-cell suspensions of spleen, MLN, and LP were extracted from naive or day 9 infected C57BL/6 WT, CD73 KO-Foxp $3^{\text {eGFP }}$, or CD4-cre TGF- $\beta$ RII ${ }^{\text {lox/lox }}$ mice and were enriched for $\mathrm{CD} 4{ }^{+} \mathrm{T}$ cells using an autoMACs (Miltenyi, Bergisch Gladbach, Germany). Cells were labeled with fluorescent dye-conjugated mAb, including anti-CD4 (RM4-5), anti-CD25 (7D4), antiCD44 (IM7), and anti-CD45RB (16 A) (all from eBioscience, San Diego, CA), and sorted by flow cytometry on a FACSAria (BD Biosciences, San Diego, CA).

Phenotypic analysis. Single-cell suspensions were incubated with anti-Fc $\gamma$ III/II receptor antibody (93) and stained with fluorochromeconjugated mAb against TCR- $\beta$ chain (H57-597), CD4 (RM4-5), CD73 (TY/11.8), CCR9 (CW-1.2), $\alpha 4 \beta 7$ (DATK-32). LIVE/DEAD kit (Molecular Probes, Saint-Aubin, France) was used to exclude dead cells. Intracellular staining was performed using the Foxp3 staining set (eBioscience). Foxp3, Nuclear Ki-67, and T-bet staining were performed using anti-Ki-67 (B56; BD Biosciences), anti-Foxp3 (FJK-16s), and anti-mouse/human T-bet (eBio4B10; both from eBioscience). The following isotype controls were used: rat IgG1 (eBRG1), mouse IgG2a (eBM2a), rat IgG2a (eBR2a), mouse IgG1 (MOPC-21), mouse IgG1 (P3.6.2.8.1; from eBioscience).

In vitro restimulation and intracellular cytokine staining. Complete RPMI-1640 medium containing 10\% fetal calf serum was used for in vitro restimulation. For cytokine detection, single-cell suspensions were cultured at $1 \times 10^{6} \mathrm{cells} \mathrm{ml}^{-1}$ in a 96 -well U-bottom plate and stimulated with $50 \mathrm{ng} \mathrm{ml}^{-1}$ PMA and $1 \mu \mathrm{g} \mathrm{ml}^{-1}$ ionomycin (Sigma) in the presence of brefeldin A (1/1,000; BD Biosciences). After $3 \mathrm{~h}$, samples were stained for dead cells. Intracellular staining was performed according to the $\mathrm{BD}$ Cytofix/Cytoperm kit protocol (BD Biosciences). Cells were stained with fluorochrome-conjugated antibodies against TCR- $\beta$ chain (H57-597), CD4 (RM4-5), CD73 (TY/ 11.8), IFN- $\gamma$ (XMG1.2), or isotype controls: rat IgG1 (eBRG1) in the presence of anti-Fc $\gamma$ III/II receptor. All antibodies were purchased from eBioscience.

In vitro assays. Purified $\mathrm{CD} 4{ }^{+} \mathrm{CD} 25^{-} \mathrm{T}$ lymphocytes from naive or day 9 infected C57BL/6 WT, Foxp3 ${ }^{\text {eGFP }}$ or CD4-cre TGF- $\beta$ RII ${ }^{\text {lox/lox }}$ mice were activated for $24 \mathrm{~h}$ with plastic-coated anti-CD3 mAb $\left(5 \mu \mathrm{g} \mathrm{ml}^{-1}\right)$ and soluble anti-CD28 $\mathrm{mAb}\left(1 \mu \mathrm{g} \mathrm{ml}^{-1}\right)$. Cultures were supplemented with $1 \mathrm{ng} \mathrm{ml}^{-1}$ TGF- $\beta$ (eBioscience) and/or $100 \mathrm{~nm}$ all-trans RA (Sigma). To analyze TGF- $\beta$ pathways, we used the TGF- $\beta$ receptor kinase inhibitor $(5 \mu \mathrm{M}, \mathrm{SB} 431542)$, p38 inhibitor ( $5 \mu \mathrm{M}, \mathrm{SB} 203580$ ), MAPK/ERK kinase inhibitor ( $5 \mu \mathrm{M}$, PD98059), JNK inhibitor (5 $\mu \mathrm{M}, \mathrm{SP} 600125)$, and PI3 kinase inhibitor (1 $\mu \mathrm{M}, \mathrm{LY} 294002)$. All were obtained from Sigma.

Adenosine quantification. CD4 ${ }^{+} \mathrm{T}$ lymphocytes from Foxp $3^{\text {eGFP }}$ mice were purified from naive or day 9 infected mice. $2 \times 10^{5} \mathrm{GFP}^{+/-}$ $\mathrm{T}$ lymphocytes were resuspended in $\mathrm{NaCl} 0.9 \%$ and incubated for $30 \mathrm{~min}$ in the presence of $1 \mathrm{~mm}$ AMP (Sigma). The supernatants were collected and adenosine concentration was analyzed by HPLC. 2-Chloro-adenosine was used as an internal standard (Sigma). The analyses were performed with an ÄKTA purifier HPLC (Amersham, Diegem, Belgium). Chromatic separation was achieved by using a Bondapak C18 column. A mobile phase which consisted of $10 \mathrm{mmoll}^{-1}$ $\mathrm{KH}_{2} \mathrm{PO}_{4}, 12 \%$ methanol and HPLC grade water, $\mathrm{pH} 5$ was used. Eluted adenosine was detected at $254 \mathrm{~nm}$. For the determination of adenosine in serum, dipyridamole ( $2 \mathrm{~mm})$ and EHNA ( $200 \mu \mathrm{m}$; Sigma) were added to prevent degradation or additional formation of plasma adenosine. Protein precipitation was performed using acetonitrile.

Western blot. Cells were lysed in RIPA buffer containing protease inhibitors and phosphatase inhibitors. Protein concentration was measured with the Micro BCA Protein Assay kit (Thermo Scientific, Erembodegem, Belgium) and protein was loaded onto a $12 \%$ Bis-Tris polyacrylamide gel. Gels were run in NuPAGE MES SDS Running Buffer (Life Technologies, Saint-Aubin, France). Proteins were transferred onto a polyvinylidene fluoride membrane (Amersham). Membranes were incubated in 5\% nonfat milk (Biorad, Nazareth Eke, Belgium, \#1706404) and proteins were detected with antibodies against: PhosphoSMAD3 (Cell Signaling Technology, Danvers, MA), SMAD3 (Cell Signaling Technology), and actin (Sigma-Aldrich, \#A2066).

Quantitative RT-PCR. RNA was extracted from cell lysates with TRIzol reagent, and reverse transcription of mRNA was carried out using Superscript II reverse transcriptase (Invitrogen) according to the manufacturer's instructions. Quantitative PCR was performed using a StepOne Plus system (Applied Biosystems, Gouda, The Netherlands) with Maxima SYBR Green/ROX qPCR Master Mix (Thermo Fisher Scientific). Quantification (with Ribosomal Protein L32 (RPL32) as endogenous housekeeping genes) was done using standard curves. The primers used in this work are listed in Supplementary Table 1. Levels of mRNA expression were normalized to RPL32 mRNA.

Treatment with A2A/A2B receptor agonists. C57BL/6 WT mice were inoculated orally with 40 cysts of ME-49 T. gondii. A2AR agonist (1 mg kg ${ }^{-1}$, CGS-21680, Sigma) and A2BR agonist $\left(3 \mathrm{mg} \mathrm{kg}^{-1}\right.$, BAY60-6583, R\&D Systems, Abingdon, UK) dissolved in $100 \mu \mathrm{l}$ vehicle (50\% PEG-400, 50\% water) were injected intraperitoneally from days 3 to 8 after infection. As a control, equivalent volume of dimethyl sulfoxide dissolved in $100 \mu$ l vehicle was used.

Quantification of fecal bacteria and parasite in tissues. Genomic DNA was extracted from the small intestinal luminal content, spleen, 
MLN, and LP using Wizard Genomic DNA Purification Kit (Promega, Leiden, The Netherlands). Quantitative PCR was performed as described previously. For quantification of parasite in tissues, levels of $T$. gondii genomic DNA expression were estimated on the detection of a well conserved region of the tandemly repeated gene, called B1 gene. $^{52}$ Levels of Enterobacteriaceae and T. gondii B1 genomic DNA expression were normalized to RPL32 genomic DNA level in each sample. The primers used in this work are listed in Supplementary Table 1.

Pathology assessment. Mice were killed on day 9 post oral infection with 40 cysts of $T$. gondii ME-49. Their small intestines were removed and immediately fixed in a solution containing $4 \%$ formaldehyde. Paraffinembedded sections were cut at $0.5 \mu \mathrm{m}$ and stained with hematoxylin and eosin. The ileum sections of the small intestine were blind examined by $\mathrm{Dr}$ Paul Delrée (IPG, Belgium). Inflammation was scored on the following modified scale ${ }^{36}$ of $0-5$ : 0 , within normal limits; 1 , minimal inflammatory leukocyte infiltrates multifocally or locally; 2 , mild inflammatory leukocytic and granulocytic infiltrates within the LP; 3 , mild to moderate inflammatory infiltrates in the LP and submucosa, alterations in intestinal crypts, local to diffuse thickening of the mucosal epithelium; 4 , moderate to marked inflammatory infiltrates of primarily viable and degenerate neutrophils, eosinophils, lymphocytes, plasma cells admixed with cellular debris within the LP and submucosa with separation or loss of crypts; 5, marked to severe inflammation diffusely with altered or complete loss of normal histologic structures and abundant inflammatory infiltrates that may extend transmurally (with peritonitis). Sections of livers were evaluated for the numbers of inflammatory loci and necrosis. Liver alanine aminotransferase levels were measured in serum samples, using commercially available kits (Roche). Serum endotoxin levels were estimated using the Limulus Amebocyte Lysate QCL-1000 assay kit (Lonza, Basel, Switzerland) according to the manufacturer's instructions.

Statistical analysis. Statistical analyses were performed using Prism6 (GraphPad Software, San Diego, CA). Statistical significance was determined with the Mann-Whitney $U$-test for two-tailed data or the Kruskal-Wallis test followed by selected comparison by Dunn's multiple comparison tests. Data are shown as median \pm interquartile range. ${ }^{\star} P<0.05,{ }^{* *} P<0.01$, and ${ }^{* * *} P<0.001$.

Ethics information. The experiments were carried out in accordance with the relevant European laws and institutional guidelines. We received specific approval for this study from the Animal Care and Use Committee of the Institute for Molecular Biology and Medicine (IBMM, ULB; protocol number CEBEA51).

SUPPLEMENTARY MATERIAL is linked to the online version of the paper at http://www.nature.com/mi

\section{ACKNOWLEDGMENTS}

We thank Oberdan Leo (ULB, Gosselies) for interesting discussion and useful suggestions; Marie-Aline Laute for her technical assistance in the performance of HPLC; Christophe Vanhaver, Jean De Clercq and Sébastien Denanglaire for their technical assistance; Adrian Liston (KUL, Leuven) for providing TGF- $\beta$ RIII/f/fl mice; Linda F. Thompson (University of Oklahoma) for providing CD73 KO mice; Stanislas Goriely (ULB, Gosselies) for providing STA1 KO mice; Caroline Abdelaziz, Philippe Veirman and Véronique Dissy for animal care. The Laboratory of Immunobiology is supported by grants of the Fonds National de la Recherche Scientifique, Wallonia (Programmes d'excellence), the Interuniversity Attraction Pole Programme and the Fonds Jean Brachet.

\section{AUTHOR CONTRIBUTIONS}

V.F., H.S., and G.O. designed the study, performed the experiments, and analyzed the data. V.A. and N.P. performed some experiments. G.O. and M.M. supervised the study. G.O., V.F., and M.M. wrote the paper. M.M. obtained the funding.

\section{DISCLOSURE}

The authors declared no conflict of interest.

(c) 2015 Society for Mucosal Immunology

\section{REFERENCES}

1. Friedman, D.J. et al. From the Cover: CD39 deletion exacerbates experimental murine colitis and human polymorphisms increase susceptibility to inflammatory bowel disease. Proc. Natl. Acad. Sci. USA 106, 16788-16793 (2009).

2. Haskó, G. et al. Ecto-5'-nucleotidase (CD73) decreases mortality and organ injury in sepsis. J. Immunol. 187, 4256-4267 (2011).

3. Bynoe, M.S. et al. CD73 is critical for the resolution of murine colonic inflammation. J. Biomed. Biotechnol. 2012, 260983 (2012).

4. Wang, L. et al. Graft-versus-host disease is enhanced by selective CD73 blockade in mice. PLoS One 8, e58397 (2013).

5. Antonioli, L., Pacher, P., Vizi, E.S. \& Haskó, G. CD39 and CD73 in immunity and inflammation. Trends Mol. Med. 19, 355-367 (2013).

6. Sprague, R.S., Ellsworth, M.L., Stephenson, A.H. \& Lonigro, A.J. ATP: the red blood cell link to NO and local control of the pulmonary circulation. Am. J. Physiol. 271, H2717-H2722 (1996).

7. Ayna, G. et al. ATP release from dying autophagic cells and their phagocytosis are crucial for inflammasome activation in macrophages. PLoS One 7, e40069 (2012).

8. Martins, I. et al. Molecular mechanisms of ATP secretion during immunogenic cell death. Cell Death Differ. 21, 79-91 (2014).

9. Beigi, R., Kobatake, E., Aizawa, M. \& Dubyak, G.R. Detection of local ATP release from activated platelets using cell surface-attached firefly luciferase. Am. J. Physiol. 276, C267-C278 (1999).

10. Ivanova, E.P., Alexeeva, Y.V., Pham, D.K., Wright, J.P. \& Nicolau, D. V ATP level variations in heterotrophic bacteria during attachment on hydrophilic and hydrophobic surfaces. Int. Microbiol. 9, 37-46 (2006).

11. Atarashi, $\mathrm{K}$. et al. ATP drives lamina propria $\mathrm{T}(\mathrm{H}) 17$ cell differentiation. Nature 455, 808-812 (2008).

12. Gombault, A., Baron, L. \& Couillin, I. ATP release and purinergic signaling in NLRP3 inflammasome activation. Front. Immunol. 3, 414 (2012).

13. Chen, Y. et al. ATP release guides neutrophil chemotaxis via P2Y2 and A3 receptors. Science 314, 1792-1795 (2006).

14. Ma, Y. etal. Anticancer chemotherapy-induced intratumoral recruitment and differentiation of antigen-presenting cells. Immunity 38, 729-741 (2013).

15. Ma, Y. et al. ATP-dependent recruitment, survival and differentiation of dendritic cell precursors in the tumor bed after anticancer chemotherapy. Oncoimmunology 2, e24568 (2013).

16. Kurashima, Y. et al. Extracellular ATP mediates mast cell-dependent intestinal inflammation through P2X7 purinoceptors. Nat. Commun. 3, 1034 (2012).

17. Schenk, U. et al. Purinergic control of T cell activation by ATP released through pannexin-1 hemichannels. Sci. Signal. 1, ra6 (2008).

18. Woehrle, T. et al. Pannexin-1 hemichannel-mediated ATP release together with P2X1 and P2X4 receptors regulate T-cell activation at the immune synapse. Blood 116, 3475-3484 (2010).

19. Schenk, U. et al. ATP inhibits the generation and function of regulatory T cells through the activation of purinergic P2X receptors. Sci. Signal. 4, ra12 (2011).

20. Haskó, G. \& Cronstein, B.N. Adenosine: an endogenous regulator of innate immunity. Trends Immunol. 25, 33-39 (2004).

21. Panther, E. et al. Adenosine affects expression of membrane molecules, cytokine and chemokine release, and the T-cell stimulatory capacity of human dendritic cells. Blood 101, 3985-3990 (2003).

22. Schnurr, M. et al. Role of adenosine receptors in regulating chemotaxis and cytokine production of plasmacytoid dendritic cells. Blood 103, 1391-1397 (2004).

23. Li, L. et al. Dendritic cells tolerized with adenosine A2AR agonist attenuate acute kidney injury. J. Clin. Invest. 122, 3931-3942 (2012).

24. Linden, J. \& Cekic, C. Regulation of lymphocyte function by adenosine. Arterioscler. Thromb. Vasc. Biol. 32, 2097-2103 (2012).

25. Csóka, B. et al. Adenosine A2A receptor activation inhibits T helper 1 and Thelper 2 cell development and effector function. FASEB J. 22, 3491-3499 (2008). 
26. Ohta, A. et al. A2A adenosine receptor protects tumors from antitumor T cells. Proc. Natl. Acad. Sci. USA 103, 13132-13137 (2006).

27. Ernst, P.B., Garrison, J.C. \& Thompson, L.F. Much ado about adenosine: adenosine synthesis and function in regulatory T cell biology. J. Immunol. 185, 1993-1998 (2010).

28. Deaglio, S. et al. Adenosine generation catalyzed by CD39 and CD73 expressed on regulatory $T$ cells mediates immune suppression. J. Exp. Med. 204, 1257-1265 (2007).

29. Borsellino, G. et al. Expression of ectonucleotidase CD39 by Foxp3 + Treg cells: hydrolysis of extracellular ATP and immune suppression. Blood 110, 1225-1232 (2007).

30. Alam, M.S. et al. CD73 is expressed by human regulatory Thelper cells and suppresses proinflammatory cytokine production and Helicobacter felisinduced gastritis in mice. J. Infect. Dis. 199, 494-504 (2009).

31. Chalmin, F. et al. Stat3 and Gfi-1 transcription factors control Th17 cell immunosuppressive activity via the regulation of ectonucleotidase expression. Immunity 36, 362-373 (2012).

32. Iyer, S.S. et al. Identification of novel markers for mouse CD4(+) T follicular helper cells. Eur. J. Immunol. 43, 3219-3232 (2013).

33. Regateiro, F.S. et al. Generation of anti-inflammatory adenosine by leukocytes is regulated by TGF- $\beta$. Eur. J. Immunol. 41, 2955-2965 (2011).

34. Iwata, M. et al. Retinoic acid imprints gut-homing specificity on T cells. Immunity 21, 527-538 (2004).

35. Gazzinelli, R.T. et al. In the absence of endogenous IL-10, mice acutely infected with Toxoplasma gondii succumb to a lethal immune response dependent on CD4 $+\mathrm{T}$ cells and accompanied by overproduction of IL-12, IFN-gamma and TNF-alpha. J. Immunol. 157, 798-805 (1996).

36. Oldenhove, G. et al. Decrease of Foxp3 + Treg cell number and acquisition of effector cell phenotype during lethal infection. Immunity 31, 772-786 (2009).

37. Louis, N.A. et al. Control of IFN-alphaA by CD73: implications for mucosal inflammation. J. Immunol. 180, 4246-4255 (2008).

38. Sun, C.-M. et al. Small intestine lamina propria dendritic cells promote de novo generation of Foxp3 T reg cells via retinoic acid. J. Exp. Med. 204, 1775-1785 (2007).

39. Coombes, J.L. et al. A functionally specialized population of mucosal CD103 + DCs induces Foxp3 + regulatory T cells via a TGF-beta and retinoic acid-dependent mechanism. J. Exp. Med. 204, 1757-1764 (2007).
40. Molenaar, R. et al. Expression of retinaldehyde dehydrogenase enzymes in mucosal dendritic cells and gut-draining lymph node stromal cells is controlled by dietary vitamin A. J. Immunol. 186, 1934-1942 (2011).

41. Mucida, D. et al. Reciprocal $\mathrm{TH} 17$ and regulatory $\mathrm{T}$ cell differentiation mediated by retinoic acid. Science 317, 256-260 (2007).

42. Odashima, M. et al. Activation of A2A adenosine receptor attenuates intestinal inflammation in animal models of inflammatory bowel disease. Gastroenterology 129, 26-33 (2005).

43. Li, Y. et al. Adenosine A2A receptor activation reduces recurrence and mortality from Clostridium difficile infection in mice following vancomycin treatment. BMC Infect. Dis. 12, 342 (2012).

44. Yegutkin, G.G. et al. Disordered lymphoid purine metabolism contributes to the pathogenesis of persistent Borrelia garinii infection in mice. J. Immunol. 184, 5112-5120 (2010).

45. Morello, S., Sorrentino, R. \& Pinto, A. Adenosine A2a receptor agonists as regulators of inflammation: pharmacology and therapeutic opportunities. J Recept. Ligand Channel Res., 11-17 (2009).

46. Ohta, A. et al. The development and immunosuppressive functions of $\mathrm{CD} 4(+) \mathrm{CD} 25(+)$ FoxP3(+) regulatory T cells are under influence of the adenosine-A2A adenosine receptor pathway. Front. Immunol. 3, 190 (2012).

47. Hart, M.L., Jacobi, B., Schittenhelm, J., Henn, M. \& Eltzschig, H.K. Cutting edge: $\mathrm{A} 2 \mathrm{~B}$ adenosine receptor signaling provides potent protection during intestinal ischemia/reperfusion injury. J. Immunol. 182, 3965-3968 (2009).

48. Madara, J.L. et al. $5^{\prime}$-adenosine monophosphate is the neutrophil-derived paracrine factor that elicits chloride secretion from T84 intestinal epithelial cell monolayers. J. Clin. Invest. 91, 2320-2325 (1993).

49. Mahamed, D.A., Mills, J.H., Egan, C.E., Denkers, E.Y. \& Bynoe, M.S. CD73-generated adenosine facilitates Toxoplasma gondii differentiation to long-lived tissue cysts in the central nervous system. Proc. Natl. Acad. Sci. USA 109, 16312-16317 (2012).

50. Raetz, M. et al. Parasite-induced TH1 cells and intestinal dysbiosis cooperate in IFN- $\gamma$-dependent elimination of Paneth cells. Nat. Immunol. 14, 136-142 (2013).

51. Hand, T.W. et al. Acute gastrointestinal infection induces long-lived microbiota-specific T cell responses. Science 337, 1553-1556 (2012).

52. Burg, J.L., Grover, C.M., Pouletty, P. \& Boothroyd, J.C. Direct and sensitive detection of a pathogenic protozoan, Toxoplasma gondii, by polymerase chain reaction. J. Clin. Microbiol. 27, 1787-1792 (1989). 